\title{
Rádios Definidos por Software com aplicações GNU Radio
}

\author{
Sérgio Bimbi Jr, Vitor C. Oliveira e Gunnar Bedicks Jr; Universidade Presbiteriana Mackenzie
}

\begin{abstract}
Abstract - The Software Defined Radio or SDR, it is a radio communication system where components which are usually implemented in hardware ( mixers, filters, amplifiers, modulators / demodulators, detectors) are implemented by software on a computer. This feature becomes important once these elements can be reconfigured and adapted to the system's conditions and preferred performance. For this application the GNU Radio / GNU Radio Companion, a free software tool that allows the developer the ability of building a SDR simply and quickly, provides a blocks building engineering with deterministic functions that facilitate integration and control parameters. This paper aims to exemplify the use and operation of SDR via GNU Radio.
\end{abstract}

Key Words-SDR, QPSK, ASK, FSK, Software Radio, GNU Radio, GNU Radio Companion, Digital Communication.

Sérgio Bimbi Junior (e-mail: sergio.bimbi@fatec.sp.gov.br) é mestrando em Engenharia Elétrica(Mackenzie), possui graduação em Engenharia de Telecomunicações pelo Centro Universitário FIEO. Atualmente é Engenheiro Eletrônico na indústria Masipack e Professor Universitário na Faculdade de Tecnologia de São Paulo(FATEC). Tem experiência na área de Engenharia Elétrica, com ênfase em Eletrônica Embarcada e Telecomunicações, desenvolvendo hardware para comandos numéricos, automação industrial e programação em VHDL. Desenvolveu trabalhos de pesquisa no Centro Universitário UNIFIEO com FPGAs que são atualmente usados em diversos trabalhos subsequentes. Possui conhecimentos desenvolvendo sistemas de medição, pesagem de balanças automáticas, programação em PLC, sistemas de controle, checadores de pesos, filtros digitais para área de medição, etc. Desenvolve pesquisa na área de SDR (QPSK) com circuitos integrados da família ZYNQ com reconfiguração parcial dinâmica.

Vitor Chaves de Oliveira (e-mail: vitor.c.br@ieee.org) é doutorando em Engenharia Elétrica (Mackenzie), Mestre em Engenharia Elétrica (PUCC), cursando Especialização em Engenharia Eletrotécnica e Sistemas de Potência (UNISAL), formado em Ciência da Computação (UNISAL) e técnico em Informática (SENAI). Professor Universitário atuando nas áreas de Eng. Elétrica e Computação; Editor de Revistas para a Sociedade Brasileira de Engenharia de Televisão (SET); Consultor em Infraestrutura TI/ERP/Telecom na Coach IT Consultoria. É autor de livro na área de computação em nuvem e já publicou dezenas de artigos em periódicos científicos e em conferências nacionais e internacionais. Seus interesses de pesquisa estão concentrados em Telecomunicações: Redes IP, Transmissões Rádio (TV Digital), Teoria Eletromagnética, Algoritmos de Controle e Modelagem Matemática de Sistemas.

Gunnar Bedicks (e-mail: gbedicks@ieee.org) é pesquisador na área de Comunicação Digital, com suas pesquisas focadas em TV digital, rádio digital e internet. Na última década ele visitou 19 países ao redor do mundo, discutindo as tecnologias para a transição da TV analógica para a TV digital. Em 2013, a Presidente do Brasil, Dilma Roussef, condecorou o Prof. Bedicks pelos serviços prestados ao país, com a mais alta honraria da diplomacia Brasileira, a medalha da Ordem do Rio Branco. Além de ser o Pesquisador Chefe do Laboratório de Pesquisas em TV Digital da Universidade Presbiteriana Mackenzie em São Paulo, ele ministra aulas de Comunicações Digitais, Sistemas de Comunicações e Sistemas de TV, para os cursos de Engenharia Elétrica na pós-graduação (mestrado e doutorado) e graduação. Ele também foi o coordenador do grupo de transmissão e modulação durante o desenvolvimento do Sistema Brasileiro de TV Digital. Atualmente coordena o GT01 - Transmissão, do Fórum do Sistema Brasileiro de TV Digital. Suas publicações incluem dezenas de artigos científicos, já tendo sido palestrante

\section{INTRODUÇÃO}

Os Rádios Definidos por Software (Software Defined Radio - SDR), são um conjunto de tecnologias implementadas em hardware e software (1), dentre estas tecnologias as aplicações em software de um Rádio Definido por Software são implementadas de maneira parcial ou total de forma reconfigurável (10) (23). Esta funcionalidade se aplica em circuitos integrados com capacidade de programação como o Field Programmable Gate Array (FPGA) e Advanced Reduced Instruction set Computer (RISC) Machine (ARM). Existem outras linhas de pesquisa sobre o critério que se aplica para definir um SDR, neste aspecto pode ser citado (2) que um SDR é um sistema de comunicação de rádio em que os componentes que eram implementados em hardware como filtros digitais, moduladores, demodulares estes passam a ser implementados em software utilizando computadores pessoais ou sistemas embarcados (23).

Os militares dos EUA foram os primeiros a aplicar rádios flexíveis em uma larga escala (2). De forma geral isso se deve a necessidade de garantir a interoperabilidade de rádios militares com outras agências governamentais como bombeiros, polícia e agências de inteligência. Isto ocorreu em virtude de cada agência exercer as suas aquisições de maneira independente, desta forma as tecnologias empregadas podem ser diferentes e incompatíveis, vislumbrando aspectos funcionais de suma importância como frequência de operação do sistema, tecnologia de modulação, dentre outros. Desta forma o padrão designado como "Rádio Digital" se aplicou para a definição de rádios com a capacidade de se adaptar a diferentes padrões de operação. Aplicando este conceito Joseph Mitola propôs em sua tese de Doutorado o conceito de rádios cognitivos (9). Este é um conceito de suma importância no cenário de aplicações SDR, o seu conceito central diz os rádios se adaptam a sua operação com a base visualizando as condições gerais do ambiente, isto visa o aprendizado de condições já passadas melhorando seu desempenho em condições futuras (3). O conceito de rádios cognitivos é muito mais amplo que o conceito aplicado a rádios digitais em virtude de aplicar técnicas de inteligência artificial, esta capaz

em diversas conferências acadêmicas, científicas e técnicas no Brasil, Estados Unidos, Argentina, Chile, Uruguai, Bolívia, Paraguai, Equador, Peru, Costa Rica, Cuba, Alemanha, Itália, Botswana, África do Sul, Ilhas Maurício e Lesotho. Sua formação inclui o título de Doutor em Engenharia Elétrica e MBA em Administração pela Universidade de São Paulo, assim como o título de Engenheiro Elétrico pelo Instituto Nacional de Telecomunicações INATEL, em Santa Rita do Sapucaí, MG. Ele detém cinco patentes e é fluente em Inglês e Alemão. 
de identificar as condições atuais e desta forma readequar o sistema a cada momento de maneira adequada. Em virtude de necessitar de alta capacidade de processamento o rádio cognitivo não recebeu grande notoriedade, ocorrido isto na ausência de tecnologias compatíveis para implementação de tal tecnologia até então. Em meados do ano de 2002 o órgão de regulador das telecomunicações dos EUA Federal Communications Commission (FCC) obteve interesse no conceito de rádios cognitivos. $\mathrm{O}$ órgão $\mathrm{FCC}$ exerceu pesquisa e veio a publicar um relatório que descrevia a utilização do espectro de rádio frequência nos EUA (4). Este relato concluía que o espectro eletromagnético estava sendo utilizado de maneira ineficiente e escasso. O FCC veio a concluir que a grande parte do espectro utilizado já obtinha um uso licenciado e os detentores destas licenças não utilizavam o espectro em tempo integral. Visualizando este cenário o FCC exerceu uma proposta de utilização do espectro de maneira oportunista, com isto os usuários poderiam utilizar o espectro quando o mesmo não estivesse sendo utilizado pelo usuário cadastrado. A condição fundamental para implementação de um rádio cognitivo é a necessidade de ter transceptores sem fio que exercem suas funções em frequências múltiplas, tão como detectar os usuários que são licenciados. Estes rádios devem ser capazes de se comunicar com diversas tecnologias de comunicação digital, aplicando esta capacidade para detecção de usuários licenciados ou trabalhar em caracterizações e frequências diferenciadas (13). Além dessas preocupações, adiciona-se a crescente preocupação com a Qualidade (24) e Disponibilidade (21) de Serviço Qos em redes de telecomunicações em geral.

\section{ARQUITETURA GERAL DE UM SDR BASEADA EM FPGAS}

Um SDR basicamente se caracteriza como uma arquitetura capaz de suportar diferentes métodos de comunicação, ajustes de canal, implementação de novas funções (12). Para execução das funções citadas outrora é necessário reprogramar o sistema na memória deum dispositivo dotado de inteligência programável como processadores FPGAs (4) (11). O processador é responsável pelo tratamento digital do esquema de modulação (18). Ele também executa a interação com os conversores Analog to Digital (ADC) e Digital to Analog (DAC) que estão conectados a um Front End $R F$, para a execução do deslocamento de frequência de transmissão e recepção. A arquitetura geral de um SDR pode ser representada pela Figura 1 .

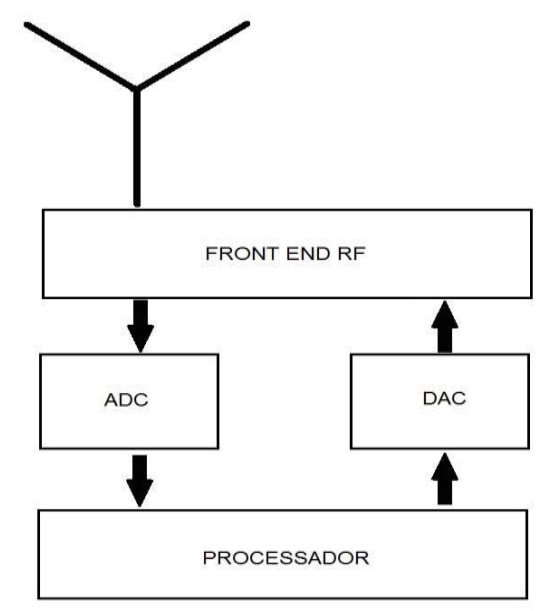

Figura 1. Arquitetura Geral de um SDR.

\section{SDR EM APLICAÇÕES COMERCIAIS}

Os SDRs estão sendo aplicados nos dias atuais afim de viabilizar a diminuição de custos em operadoras de telecomunicações (14) (16). Estão presentes no mercado também empresas que exploram mercados como o de rádio amadores. Algumas aplicações em rádios amadores que necessitam desenvolver suas próprias técnicas de recepção para aplicações como a de rádios amadores ou de uso geral. Em algumas aplicações rádios amadores são alocados em receptores SDR conectados à internet, estas aplicações possibilitam difundir o SDR para que qualquer usuário comum possa sintonizar frequências ao redor do mundo sem que tenha a necessidade de custos com um hardware específico de um SDR. Na atualidade o SDR é uma tecnologia amplamente difundida, com grande disponibilidade de plataformas sem custo (4). Existem uma gama de softwares livres (22) (23), com um bom suporte pra integração em ambientes de desenvolvimento. Entretanto as plataformas atuais necessitam de evolução para alcançar requisitos de alto desempenho como largura de banda e eficiência de energia (14) (23) (25), estes citados são necessários para se equiparar a sistema de alta capacidade como o Long Term Evolution (LTE) que é um protocolo pertencente ao sistema 4G e o IEEE 802.1 1ac.

\section{SDR EM APLICAÇÕES ACADÊMICAS}

Nos dias atuais estão presentes no mercado diversas plataformas comercias e acadêmicas de baixo custo, estas são capazes de implementar rádios 3G e rádios IEEE 802.1 $1 \mathrm{a} / \mathrm{b} / \mathrm{g} / \mathrm{n}$ e outras tecnologias. Com isto diversos grupos de pesquisa surgiram afim de integrar o SDR em suas linhas de pesquisa e desenvolvimento. Um dos principais aspectos ligados ao SDR e que ele diminuiu em larga escala o custo de pesquisa voltadas a camada física e enlace. Com isto as pesquisas migraram de grandes centros de e pesquisa dotados da capacidade de investimento em pesquisa para centros que disponibilizam menores capacidade financeira. Dentre as linhas de pesquisa pode ser citado o uso de codificação de rede, afim de aumentar a capacidade, recuperação de quadros 
perdidos em processamento digital de sinais, códigos Rateless, rádios full-duplex.

\section{COMUNICAÇÃO DIGITAL}

Em aspectos funcionais pode-se modelar um sistema de comunicação digital de dados em banda passante como é demonstrado na Figura 2. A princípio se supõe que exista uma fonte de mensagem capaz de emitir símbolos a cada $T$ segundos. Estes símbolos são pertencentes a um alfabeto de $M$ símbolos, os quais podem ser visualizados na Equação 1 (5).

$$
m 1, m 2, \ldots, m n(1)
$$

As probabilidades dos símbolos, a priori, podem ser demonstradas pela Equação 2 (5).

$$
P(m 1), P(m 2), \ldots, P(m n)(2)
$$

Estas probabilidades especificam a saída de uma fonte de mensagem, ou seja, quando $M$ símbolos de um alfabeto são igualmente prováveis é obtida a probabilidade que pode ser vista pelas equações 3 e 4 .

$$
\begin{aligned}
& p i=P(m 1)(3) \\
= & \frac{1}{M} \text { para todo } i(4)
\end{aligned}
$$

A saída $M$ primária da fonte de mensagem é apresentada a um codificador de transmissão de sinal, este produz um vetor correspondente $s$, que são compostos de $N$ elementos reais, produz também, um conjunto destes elementos para cada $M$ símbolos do alfabeto fonte; a dimensão $N$ é menor ou igual a $M$. Tendo o vetor $s$ como entrada, o modulador constrói um sinal distinto $s 1(t)$ de duração $T$ segundos com a representação do símbolo $m$ gerado pela fonte de mensagem. O sinal $s 1(t)$ é necessariamente um sinal de energia, como pode ser visto pela Equação 5 (5).

$$
E i=\int_{0}^{t} s_{i}^{2}(t) d t i=1,2, \ldots, M(5)
$$

Logo, s1(t) é um valor real. Um desses sinais é transmitido a cada $T$ segundos. $O$ sinal específico escolhido para a transmissão depende da mensagem de entrada e, possivelmente dos sinais transmitidos em intervalos de tempo precedente. Com uma portadora senoidal, o recurso utilizado pelo modulador para distinguir um sinal do outro é uma mudança de passo na amplitude, frequência ou fase da portadora. Também pode ser utilizada uma forma híbrida de modulação que combine mudanças de amplitude e fase de amplitude ou de frequência.

Os sinais aplicados em sistemas digitais são valores binários únicos (0 e 1), esses são definidos com a tensão de saída de uma quantização de volts para o valor mínimo e outra quantização para o valor máximo. Se for implementada uma modulação a sinais caracterizados neste padrão, o sistema estará sujeito à sensibilidade a ruído, quando o ruído interfere no sinal modulado pode ser interpretado um pulso, que mude o conteúdo da sua informação. $\mathrm{O}$ chaveamento de fase coerente está disposto sobre a detecção coerente de sinais em presença de um ruído branco gaussiano aditivo. Dentro desse aspecto estão incluídos sistemas de transmissão de dados em banda passante de maneira específica.

Dentre os padrões destacam-se os sistemas Amplitude Shift Keying (ASK), Frequency Shift Keying (FSK), Phase Shift Keying (PSK), Quadrature Phase Shift Keying (QPSK) e suas variantes.

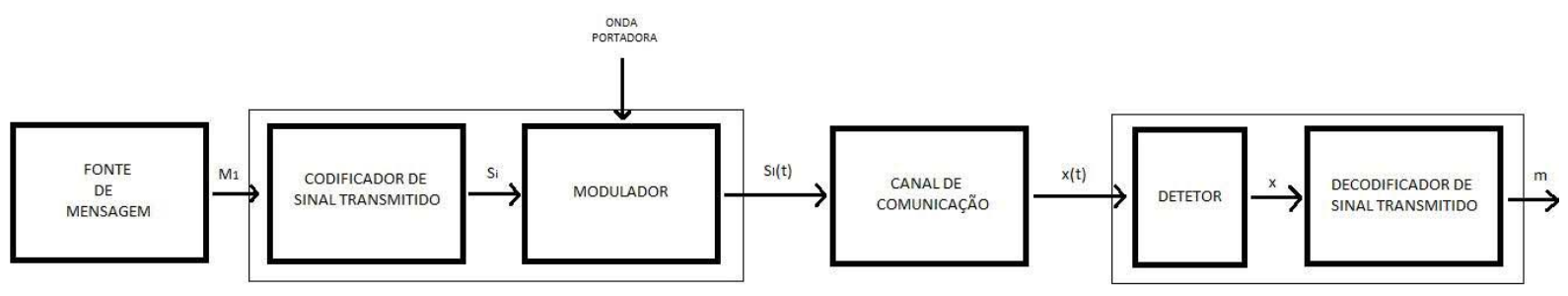

Figura 2. Modelo funcional de um sistema de comunicação digital de dados em banda passante.

\section{MODULAÇÃO ASK}

A modulação ASK se consiste na variação da amplitude da portadora senoidal, mediante ao estado lógico do sinal do modulador. Esta técnica, compreendida pela Figura 3, constituiu um modulador gerando padrões binários, combinado com uma portadora fixa. Quando o modulador está em nível lógico 1 conduz o estado atual da portadora:
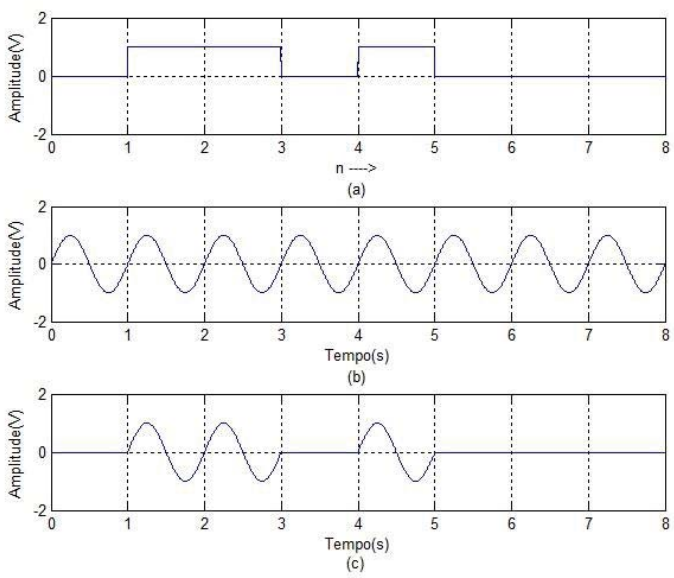

Figura 3. Representação da forma de onda de um modulador ASK: Entrada Bit Stream (a), Portadora(b), Forma de onda ASK(c). 


\section{MODULAÇÃO FSK}

A modulação FSK gera a modificação na frequência da portadora senoidal, esta modificação _e realizada mediante as variações dos estados lógicos do sinal modulador. Na Figura 4 pode-se entender o funcionamento de um sistema FSK com a visualização do sinal binário gerado pelo modulador, o sinal transmitido com técnica de modulação aplicada e o sinal reconstituído.

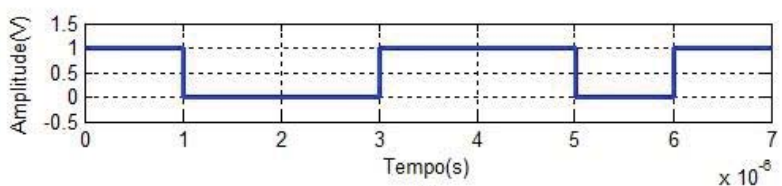

(a)
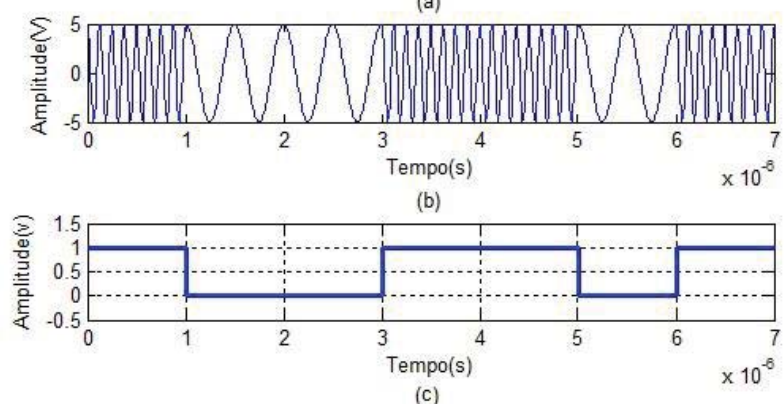

(c)

Figura 4. Representação da forma de onda de um modulador FSK: Sinal Digital (a); Forma de onda FSK correspondente à informação binária(b); Informação digital recebida após a demodulação FSK(c).

\section{MODULAÇÃO PSK}

Em um modulador PSK binário coerente o par de sinais f1(t) e f2(t)_e utilizado para representar os símbolos binários 1 e 0, esses pares podem ser vistos pelas Equações 6 e 7(5).

$$
\begin{gathered}
s_{1} t=\sqrt{\frac{2 E_{b}}{T_{b}}} \cos \left(2 \pi f_{c} t\right)(6) \\
s_{2} t=\sqrt{\frac{2 E_{b}}{T_{b}}} \cos \left(2 \pi f_{c} t+\pi\right)=-\sqrt{\frac{2 E_{b}}{T_{b}}} \cos \left(2 \pi f_{c} t\right)(7)
\end{gathered}
$$

Onde $0 \leq t \leq T b E b$ é a energia do sinal por bit transmitido. Para assegurar que cada bit transmitido contenha um número integral de ciclos da onda portadora, a frequência portadora $F C$ é escolhida de maneira igualada a $n c / T b$ para algum número fixo de $n c$. Um par de ondas senoidais que diferem somente em um desvio de fases relativos de 180 graus, como é definido pelas Equações 9 e 10, denomina-se sinais antipodais (5). Em função desse par de equações, e restritivamente, no caso do PSK binário, há somente uma função base de energia unitária que está representada na Equação 8(5).

$$
\begin{gathered}
\phi_{1} t=\sqrt{\frac{2}{T_{b}}} \cos \left(2 \pi f_{c} t\right), 0 \leq t \leq T_{b}(8) \\
s_{1} t=\sqrt{E_{b} \phi_{1(t)}}, 0 \leq t \leq T_{b}(9) \\
s_{2} t=-\sqrt{E_{b} \phi_{1(t)}}, 0 \leq t \leq T_{b}(10)
\end{gathered}
$$

Um sistema PSK binário coerente caracteriza-se por ter um espaço de sinal unidimensional com uma constelação de sinais consistentes em dois pontos de mensagem. As coordenadas dos pontos de mensagem podem ser visualizadas pelas Equações 11, 12, 13 e 14 (5).

$$
\begin{gathered}
s_{11}=\int_{0}^{T_{b}} s_{1}(t) \phi_{1}(t) d t(11) \\
=+\sqrt{E_{b}}(12) \\
s_{21}=\int_{0}^{T_{b}} s_{2}(t) \phi_{1}(t) d t(13) \\
=-\sqrt{E_{b}}(14)
\end{gathered}
$$

A caracterização da forma de onda de um sinal PSK visualizando a entrada de dados, o sinal senoidal e o sinal PSK modulado podem ser vistos através da Figura 5.
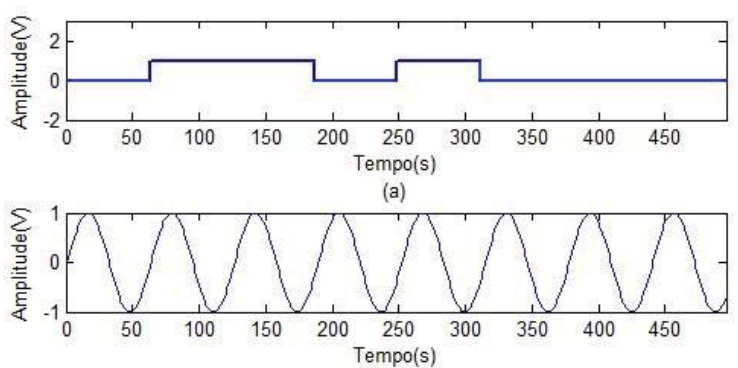

(b)

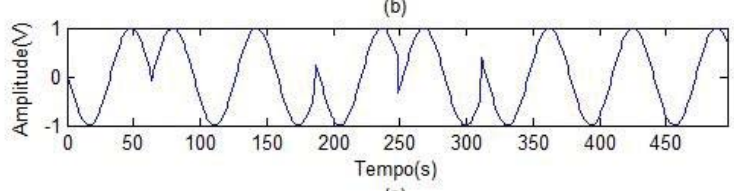

(c)

Figura 4. Representação da forma de onda de um modulador PSK: Sequência de entrada de dados (a); Sinal senoidal(b); Sinal PSK modulado(c).

\section{MODULAÇÃO QPSK}

Em um modulador QPSK dois bits sucessivos na sequência são agrupados em um símbolo. Na entrada deste modulador é realizada uma série de conversões dos bits de entrada, posterior a este processo a conversão do sinal unipolar para bipolar é realizada. Após estes processos é necessário executar a filtragem dos sinais para evitar a distorção do sinal recebido, que se caracteriza como interferência intersimbólica (ISI). Após a filtragem, o sinal modulado está pronto para aplicação. No final do processo o sinal modulado é gerado em dois ramos separados caracterizados em $I$ e $Q$. Estes sinais são resumidos e traduzidos em um sinal analógico caracterizado por um conversor digital analógico. O sinal analógico QPSK e caracterizado matematicamente pelas Equações 15, 16 e 17 (5).

$$
\begin{gathered}
s Q P S K=d I(t) \cos (2 \pi f 0 t)-d Q(t) \sin (2 \pi f 0 t) \\
d I(t)=\sqrt{\frac{2 E}{T}} \cos \left[(2 i-1) \frac{\pi}{4}\right](16) \\
d Q(t)=\sqrt{\frac{2 E}{T}} \sin \left[(2 i-1) \frac{\pi}{4}\right](17)
\end{gathered}
$$

Existem diferenças entre a implementação digital de um modulador QPSK em um FPGA no que diz respeito a sua implementação convencional em eletrônica analógica. Sendo aplicada em eletrônica convencional a sua abordagem exigiria 
a decomposição dos sistemas e subsistemas e a necessidade de mais componentes de hardware. Por exemplo, a conversão de série para paralelo exigiria um grande número de Filip Flaps $D$ e $T$. A geração da portadora exigiria o uso de um oscilador controlado por tensão (Voltagem Controle Oscilador (VCO)), além de um deslocador de fase em 90 graus. Para multiplicar a portadora e o sinal de modulação é necessário a implementação de dois mixers. Além disso, para reconstituir o sinal modulado é necessário o uso de dois filtros no mínimo. Com o uso do FPGA, o modulador QPSK poderia ser implementado quase que integralmente em um único dispositivo juntamente com um complemento indispensável que é o conversor digital analógico. Há uma vantagem bastante conveniente na implementação de sistemas utilizando dispositivos FPGA, para a implementação de um modulador QPSK é necessário implementar os blocos que desempenharão funções equivalentes as funções que são executados por circuitos convencionais. As funções do oscilador de tensão são controladas em domínio digital, onde são realizadas por oscilador de comandos numéricos (Numerically Controlled Oscillator (NCO)). O NCO, por sua vez é utilizado para sintetizar o tempo discreto a fim de caracterizar uma onda senoidal. Os filtros necessários para a caracterização do sinal (FIR), cuja a função de transferência tem a forma de cosseno levantado, também podem ser aplicados na arquitetura de um FPGA. Afim de executar a detecção de um sinal QPSK é exigido um detector coerente. Torna-se necessário, então, recuperar as referências de $\cos (\omega c t)$ e $\operatorname{sen}(\omega c t)$. Estas referências necessitam ter exatamente a mesma frequência e fase das geradoras do sinal QPSK no modulador. O diagrama de blocos da Figura 5 mostra um detector QPSK.

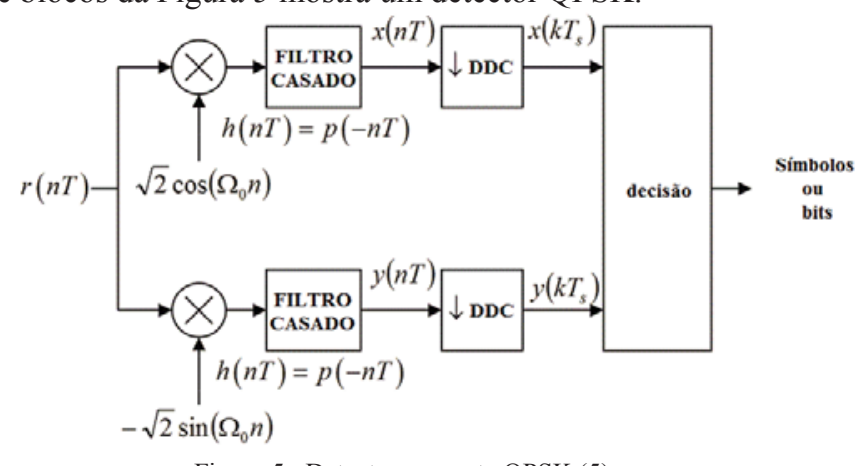

Figura 5. Detector coerente QPSK (5).

Para verificar como opera o detector, serão estabelecidas as equações trigonométricas de cada um dos 4 estados. A Figura 6 e a Tabela 1 ilustram sua equação para cada um dos estados.

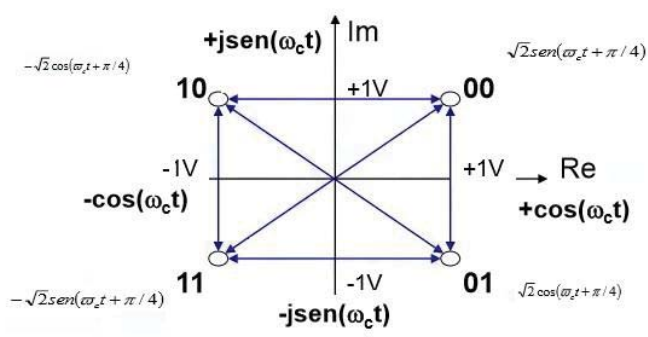

Figura 5. Constelação QPSK (6).

\begin{tabular}{|c|c|c|}
\hline \multicolumn{2}{|c|}{ Figura 5. Constelação QPSK (6). } \\
\hline Dbit Entrada & Fase do Sinal QPSK & $\begin{array}{c}\text { Coordenadas dos } \\
\text { pontos de mensagem } \\
s_{i 1} \text { e } s_{i 2}\end{array}$ \\
\hline
\end{tabular}

\begin{tabular}{|c|c|c|}
\hline 10 & $\frac{\pi}{4}$ & $+\sqrt{E / 2}-\sqrt{E / 2}$ \\
\hline 00 & $\frac{3 \pi}{2}$ & $-\sqrt{E / 2}-\sqrt{E / 2}$ \\
\hline 01 & $\frac{5 \pi}{4}$ & $-\sqrt{E / 2}+\sqrt{E / 2}$ \\
\hline 11 & $\frac{7 \pi}{4}$ & $+\sqrt{E / 2}+\sqrt{E / 2}$ \\
\hline
\end{tabular}

Tabela 1. Caracterização do espaço de sinal QPSK (17).

A Figura 6 ilustra a forma de onda de um modulador QPSK com suas sequências e pares de ondas moduladas e demoduladas.
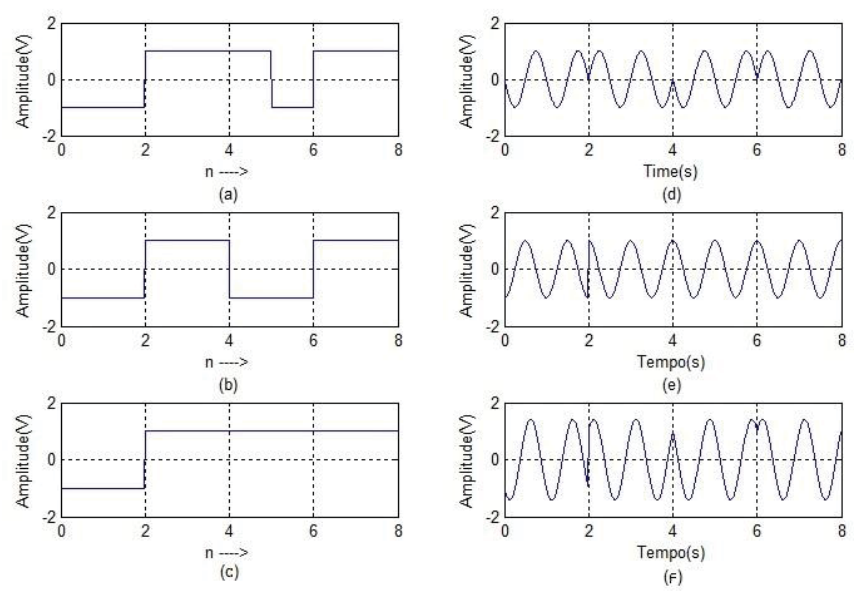

(F)

Figura 6. Representação da forma de onda de um modulador QPSK: Entrada bit Stream (a); Sequência Par(b); Sequência Impar(c); Sequência Par de Ondas Demodulada Binary Phase Shift Keying (BPSK) (d); Sequência Impar de Onda Demodulada BPSK (e) e Onda Demodulada QPSK(f)

\section{INTRODUÇÃO AO GNU RADIO}

O GNU Radio é caracterizado por um conjunto de ferramentas e códigos abertos, que oferece um ambiente de desenvolvimento de blocos de processamento capazes de implementar uma arquitetura de SDR em software. O GNU Radio possui a capacidade de integração com inúmeras plataformas de hardware. As aplicações integradas ao sistema e ambiente de desenvolvimento são desenvolvidas com a ferramenta Phython, este está incumbido de exercer a conexão dos blocos básicos de processamento vinculados ao sistema. Estes blocos são desenvolvidos na linguagem $\mathrm{C}++$, em virtude desta possibilitar maior desempenho. Também está presente no sistema um ambiente de desenvolvimento mais amigável que é o GNU Radio Companion (GRC), este permite o desenvolvimento de aplicações utilizando uma interface gráfica com blocos que exercem funções determinísticas. Para instalar o GNU Radio no Linux existem várias maneiras, uma das mais amigáveis é utilizar scripts que instalam e atualizam as dependências de forma automatizada (19).

\section{GNU RADIO COMPANION}

O GNU Radio Companion (GRC) é uma ferramenta gráfica livre que possibilita ao desenvolvedor criar protótipos de sistemas de comunicação de fácil implementação e com tempo de desenvolvimento reduzido (15). Programas que são criados no GNU Radio não precisam necessariamente ser construídos no GRC, no entanto a utilização desta ferramenta possibilita ao desenvolvedor uma rápida criação de protótipos em virtude 
de possuir blocos gráficos de fácil entendimento e integração (20).

\section{DEMODULADOR DE SINAL FREQUENCY} MODULATION (FM) COM GNU RADIO E CHIPSET RTL2832

Neste exemplo é implementado um demodulador FM. Utilizando um hardware baseado no receptor RTL2832 do fabricante Realtek, este módulo é um Digital Video Broadcasting (DVB-T), o módulo se torna interessante em virtude de poder oferecer ao desenvolvedor um SDR de baixo custo (aproximadamente U\$ $\$ 20,00$ ), possui características coerentes para implementação de um SDR, suporta modos $2 \mathrm{~K}$ e $8 \mathrm{~K}$ com 6,7 e largura de banda de $8 \mathrm{MHz}$, parâmetros de modulação como taxa de código e intervalo de guarda são detectados de forma automática, suporta módulos de
Frequência Intermediária (FI) de $36,125 \mathrm{MHz}$, FI baixa de $4,57 \mathrm{MHz}$ e FI Zero de 28,8MHZ. É capaz de suportar FM, Digital Audio Broadcasting (DAB) e DAB + suporte de rádio. Em sua arquitetura está incorporado um Analog to Digital Converter (ADC) este apresenta alta estabilidade na recepção portátil. O RLT2832 apresenta algoritmos de proprietários da Realtek incluindo estimativa de canal, interface de co-canal, recepção de canal longo com rejeição de eco de ruído impulsivo além de conexão Universal Serial Bus (USB) (8). Para implementar tal funcionalidade é construído um modelo utilizando o GNU Radio Companion, como mostra a Figura 7. O modelo desenvolvido demonstra os blocos implementados no GNU Radio Companion a fim de construir um demodulador FM. Dentro deste modelo temos os módulos:

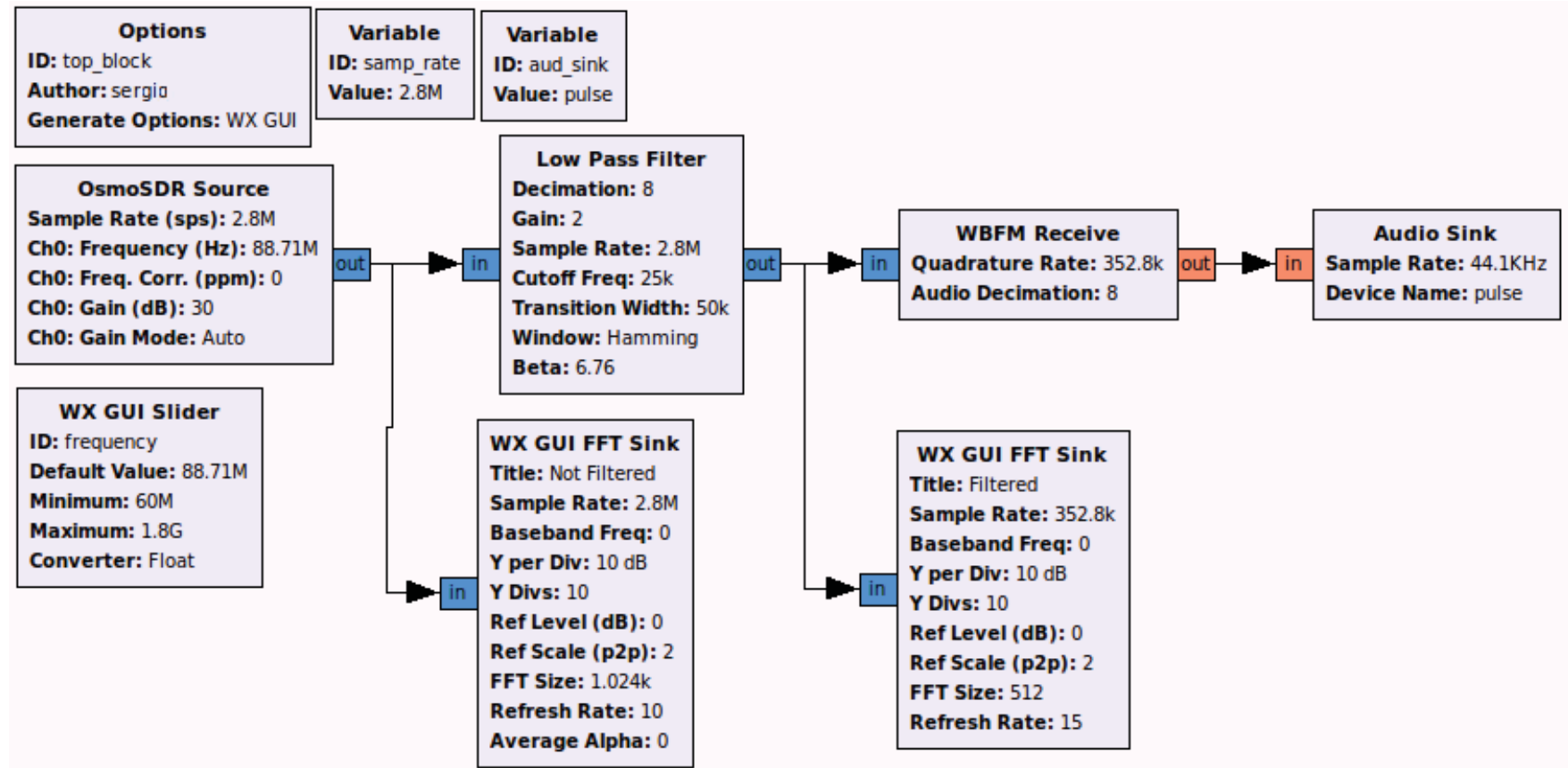

Figura 7. Modelo básico de um demodulador FM no GNU Radio Companion.

1) Options

Bloco do GRC para introdução de parâmetros de controle do projeto como, Nome do Projeto e autor.

2) Variable

Bloco do GRC para introdução variáveis que podem ser dinamicamente alteradas pelo desenvolvedor.

3) OsmoSDR Source

Bloco do GRC para captação do sinal de rádio, inserido pelo módulo com o Chipset RTL2832.

4) Low Pass Filter

Bloco do GRC para execução de um filtro passa baixa aplicado ao sinal de entrada, com decimação de 8 , ganho de 2 , taxa de amostragem de $2,8 \mathrm{MHz}$, Cutoff de $25 \mathrm{KHz}$, largura de transmissão de $50 \mathrm{Khz}$, janela de Hamming e Beta de 6,76.
5) WBFM Receive

Bloco do GRC para recepção e tratamento do sinal de rádio recebido pós filtro.

6) Audio Sink

Bloco do GRC para conexão com áudio do computador.

7) WX GUI Slider

Bloco do GRC para alteração da frequência de sintonia recebida pelo modelo proposto.

8) WX GUI FFT Sink

Bloco do GRC para alteração da frequência do espectro recebido pelo modelo proposto.

9) $\mathrm{Na}$ Figura 8 podem ser visualizados os espectros recebidos antes e depois do filtro, estes gerados pelos blocos WX GUI FFT Sink. 


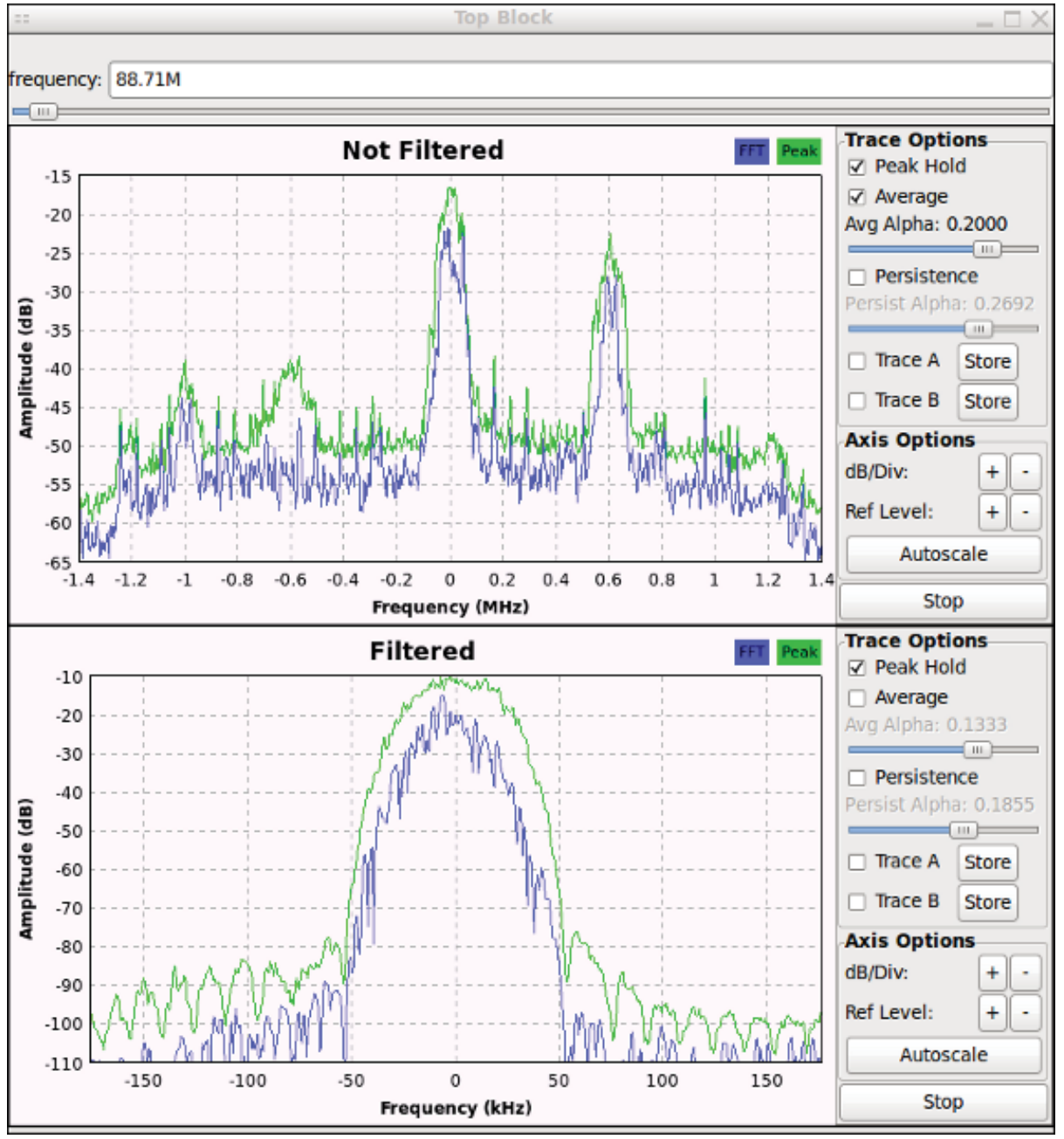

Figura 8. Espectros recebidos antes do filtro digital (Not Filtered) e depois do filtro digital (Filtered).

O modelo implementado demonstra os blocos utilizados no GNU Radio Companion a fim de construir um demodulador FM. O sistema possui o bloco de entrada baseado no hardware do Chipset receptor RTL2832 do fabricante Realtek, este responsável pela aquisição do sinal de rádio. Posterior a este processo é implementado um filtro do tipo passa baixa a fim de exercer uma melhor recepção e estabilidade. Posterior a este processo está contido o bloco receptor FM e por fim conexão ao áudio do sistema (áudio do computador).

\section{CONCLUSÃO}

O artigo apresenta uma visão ampla sobre o estado da arte de sistemas e aplicações SDR, abordando questões históricas além de aspectos voltados a aplicações comerciais e acadêmicas. Também descreve características básicas de comunicação digital em aspectos teóricos como explanação dos padrões mais importantes de modulação presentes nos dias atuais. Descreve a ferramenta livre GNU Radio e descreve um exemplo básico de criação de um SDR utilizando o hardware com Chipset RTL2832 exercendo integração com a ferramenta GNU Radio Companion, com isto oferecendo a implementação de um SDR demodulador FM de forma didática.

\section{REFERÊNCIAS}

[1] Wireless Innovation Forum. Wireless Innovation Forum Available: http://www.wirelessinnovation.org.

[2] Dillinger, M., Madani, K, and Alonistioti, N. (2003). Software Defined Radio: Architectures, Systems and Functions. Wiley \& Sons.

[3] Cognitive Radio - An Integrated Agent Architecture for Software Defined Radio. PhD thesis, Royal Institute of Technology (KTH).

[4] Force, S. P. T. (2002). Spectrum policy task force report et docket no. 02-155. Technical report, FCC.

[5] KAZAZ MERIMA KULIN, M. H. T. Design and Implementation of SDR Based QPSK Modulator on FPGA. 1. ed. Bosnia and Herzegovina: IEEE, 2013.

[6] HAYKIN, S. Sistemas de Comunicação Analógicos e Digitais. 4. ed. BRA: Bookman, 2004

[7] AKAMINE, C. Demodulação Banda Base/Passante M-PSK e M-QAM. 1. ed. BRA: Mackenzie, 2013.

[8] RTL2832U Available:

http://www.realtek.com.tw/products/productsView.aspx?Langid=1\&PFid $=35 \&$ Level $=4 \&$ Conn $=3 \&$ ProdID $=257$.

[9] Mitola III, J., 1992. Software Radios Survey, Critical Evaluation and Future Directions. IEEE National Telesystems Conference. NTC-92. ISBN: 0-7803-0554-X. p. 13/15 - 13/23. DOI: 10.1109/NTC. 1992.267870

[10] Marpanaji, E., Trilaksono, B.R., Langi, A.Z.R., Kurniawan, A., Mahendra, A. and Liung, T., 2007. Experimental Study of DQPSK Modulation on SDR Platform. Journal of ICT Research and Applications. ISSN: 2337-5787. Vol. 1, No. 2, 2007, p. 84-98.

[11] Kazaz, T., Kulin, M. and Hadzialic, M., 2013. Design and implementation of SDR based QPSK modulator on FPGA. 36th IEEE International Convention on Information \& Communication Technology 
Electronics \& Microelectronics. MIPRO. ISBN: 978-953-233-076-2. May 2013, p. 513-518.

[12] Priya, A., Rajesh, N. and Muthaiah, R., 2013. CARRIER SYNCHRONIZATION IN SOFTWARE DEFINED RADIO FOR 8PSK. International Journal of Engineering and Technology. IJET. ISSN: 0975-4024. Feb.-Mar. 2013, Vol. 5, No.1, p.81-87.

[13] Cummings, M. and Haruyama, S., 1999. FPGA in the software radio. IEEE Communications Magazine. ISSN: 0163-6804. Feb. 1999, Vol. 37, Issue 2, p. 108-112. DOI: 10.1109/35.747258

[14] Lathi, B.P. and Ding, Z., 2009. Modern Digital and Analog Communication Systems. Oxford Series in Electrical and Computer Engineering. Oxford University Press. ISBN: 9780195331455 . Fourth Edition. 1004 p.

[15] Abirami, M., Gandhiraj, R. and Soman K.P., 2013. Performance Analysis of Real Time OFDM Based Communication System Using GNU Radio and USRP. International Journal of Advanced Research in Computer Science and Software Engineering. IJARCSSE. ISSN: $2277-$ 128X. June 2013, Vol. 3, Issue 6, p. 1584-1590.

[16] Chen, K. and Duan, R. (2011). C-RAN -the road towards green RAN Technical report, China Mobile. http://abs.chinamobile.com/cran/wpcontent/uploads/CRAN_white_paper_v2_5_EN.pdf.

[17] TANENBAUM \& WETHERALL; 2010 . Computer Networks 5th Edition. New Jersey, United States of America. Publisher: Prentice Hall. 960p. ISBN: 0132126958, 978-0132126953.

[18] Rappaport, T. (2001). Wireless Communications: Principles and Practice. Prentice Hall PTR, Upper Saddle River, NJ, USA, 2nd edition.

[19] Build-Gnuradio. http://www.sbrac.org/files/build-gnuradio. Acessado em Março de 2015

[20] Out-of-tree modules. http://gnuradio.org/redmine/projects/gnuradio/wiki/OutOfTreeModules. Acessado em Março de 2015.

[21] Oliveira, V., Yano, I., Mota, A., Mota, L.; 2013. FEASIBILITY OF DESKTOP VIRTUALIZATION PER SOFTWARE SERVICES AND LOCAL HARDWARE BASED ON THE NETWORK THROUGHPUT. Journal of Computer Science. ISSN: 1549-3636. Vol. 9, Issue 7, p. 827-837. DOI: 10.3844/jessp.2013.827.837 .

[22] Oliveira, V., Bedicks Jr, G., Akamine, C.; 2015. METHODOLOGY TO ASSES IP CONNECTION AVAILABILITY: A PREREQUISITE FOR FEASIBLE VIDEO STREAM THROUGH CONTENT DELIVERY NETWORKS. Journal of Computer Science. ISSN: 1549-3636. Vol. 11 , Issue 2, p. 426-437. DOI: 10.3844/jessp.2015.426.437 .

23] Bimbi Jr, S., Oliveira, V., Bedicks Jr, G.; 2015. SOFTWARE DEFINED RADIO IMPLEMENTATION OF A OPSK MODULATOR/DEMODULATOR IN AN EXTENSIVE HARDWARE PLATFORM BASED ON FPGAs XILINX ZYNQ. Journal of Computer Science. ISSN: 1549-3636. Vol. 11, Issue 4, p. 598-611. DOI: 10.3844/jcssp.2015.598.611 .

[24] Oliveira, V., Mota, A., Mota, L; 2013. IMPACTS OF APPLICATION USAGE AND LOCAL HARDWARE ON THE THROUGHPUT OF COMPUTER NETWORKS WITH DESKTOP VIRTUALIZATION. American Journal of Applied Sciences. ISSN: 1546-9239. Vol. 10, Issue 1, p. 117-122. DOI: 10.3844/ajassp.2013.117.122 .

[25] Yano, I., Oliveira, V., Becker, M., Corrêa, A.; 2015. APPLYING A HYBRID POLLING APPROACH BY SOFTWARE IMPLEMENTATION TO EXTEND THE LIFETIME OF A WIRELESS SENSOR NETWORK. Journal of Computer Science. ISSN: 1549-3636. Vol. 11, Issue 4, p. 699-706. DOI 10.3844 /jcssp.2015.699.706

\section{Cite this article:}

Bimbi Junior, S. , Chaves de Oliveira, V., Bedicks Junior, G.; 2015.

Rádios Definidos por Software com aplicações GNU Radio. SET EXPO PROCEEDINGS. ISSN Print: 2447-0481.ISSN Online: 2447-049X. v.1.doi: 10.18580/setep.2015.1.2 . Web Link: http://dx.doi.org/10.18580/ setep.2015.1.2 